\title{
Colorimetric gas sensors for the detection of ammonia, nitrogen dioxide and carbon monoxide: current status and research trends
}

\author{
J. Wöllenstein ${ }^{1,2)}$, C. Peter ${ }^{2)}$, M. Schiel ${ }^{2)}$, K.Schmitt ${ }^{2)}$ \\ 1) University of Freiburg, George Köhler Allee 102, 79110 Freiburg \\ 2) Fraunhofer Institute for Physical Measurement, Heidenhofstr. 8, 79110 Freiburg
}

\begin{abstract}
Colorimetric detection of gases has been used for years. Colorimetric gas sensors are based on the change in color of a chemochromic reagent incorporated in a porous matrix. Several products are available on the market, such as the well established Dräger gas tubes [1]. When the gas is sucked in the tube a color change occurs and its concentration can be evaluated on a reference scale. This publication focuses on the state-of-the-art of colorimetric gas sensors and current developments for the detection of ammonia, nitrogen dioxide and carbon monoxide.

For fire detection, the detection of particularly $\mathrm{CO}$ and $\mathrm{NO}_{2}$ as leading substances is of high interest. In special applications CO-sensors are already used to support conventional optical or heat detectors for fire detection. Although $\mathrm{CO}$ is a leading gas in many fire events, there are other fire events emitting mainly $\mathrm{NO}_{2}$ (for example polyurethane or n-heptane fires). Gas sensors for the detection of $\mathrm{CO}$ or $\mathrm{NO}_{2}$ are available on the market, yet are too expensive, the dimensions are too big or the long-term stability is too poor. Additionally the power consumption of most suitable technologies is much too high. We present our work on colorimetric films combined with a MEMS-compatible low-power technology for the $\mathrm{CO}$ and $\mathrm{NO}_{2}{ }^{-}$ detection in order to overcome these drawbacks of the existing technologies.
\end{abstract}

\section{Motivation}

The advantage of colorimetric gas sensors is their high selectivity to only one gas. The selectivity is caused by a chemical reaction between gas and dye. The reaction depends on the chromogenic material. For the detection of ammonia, pH indicators like bromophenol blue or bromocresol purple can be used. In this case, the gas acts as a Lewis-base and induces the color change due to hydrogen release. Other gasochromic materials are complexes. Their color chance is induced through changes in the ligand field. The most common gas sensors working with the gasochromic principle are the so-called Dräger gas tubes. These packed glass tubes are used by fire departments in case of an unknown fire. The endings of the tube get bricked away to pump gas inside. The concentration of the detected gas can be easily read from an imprinted scale on the tube. The main disadvantage of these sensors is that always an operator is used to read out the exact gas concentration.

The work presented here offers the possibility of a standalone colorimetric gas sensor system. A simple measurement set-up enables an automatic measurement. The sensor is based on a planar optical waveguide. In the easiest case this can be a microscope slide. The light of an LED is coupled into one end of this waveguide and passes through it under the conditions of total inner reflection (TIR). After decoupling at the opposite side, the light is focused on a photo detector. The dye can be deposited onto this waveguide by dip- or spin coating. To obtain homogeneous films, the dye is embedded into a polymeric matrix. These polymers should offer the following properties: long term stability, no swelling due to humidity and minimal interference with the dye. Possible polymers are poly vinyl chloride (PVC), ethyl cellulose (EC) and/or poly methylmethacrylate (PMMA). The color change of the dye, due to gas exposure, leads to changes in the evanescent field on the waveguide surface. These changes can be directly detected by changes in the output voltage of the photo detector. The working principle of this sensor is shown in Figure 1. 


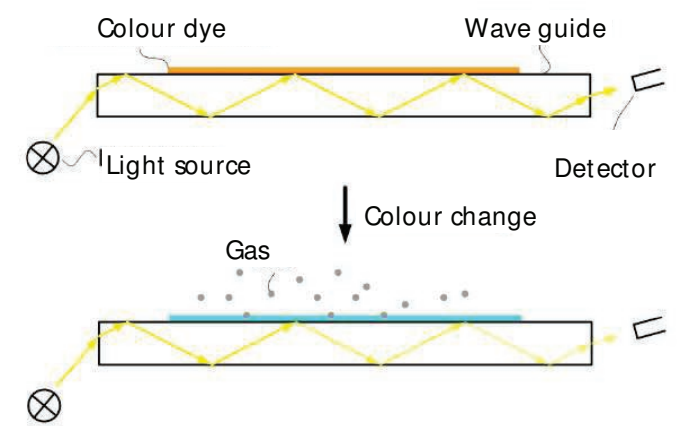

Figure 1: Picture of the of microscope slide with the lower half covered with the chemochromic film. The light of an LED is coupled into one end of the waveguide and travels trough it under TIR before it is focused onto a photodetector. Above: before gas exposure. Down: schematic cross-sectional view after gas exposure and induced color change.

A picture of the developed measurement chamber is shown in Figure 2.

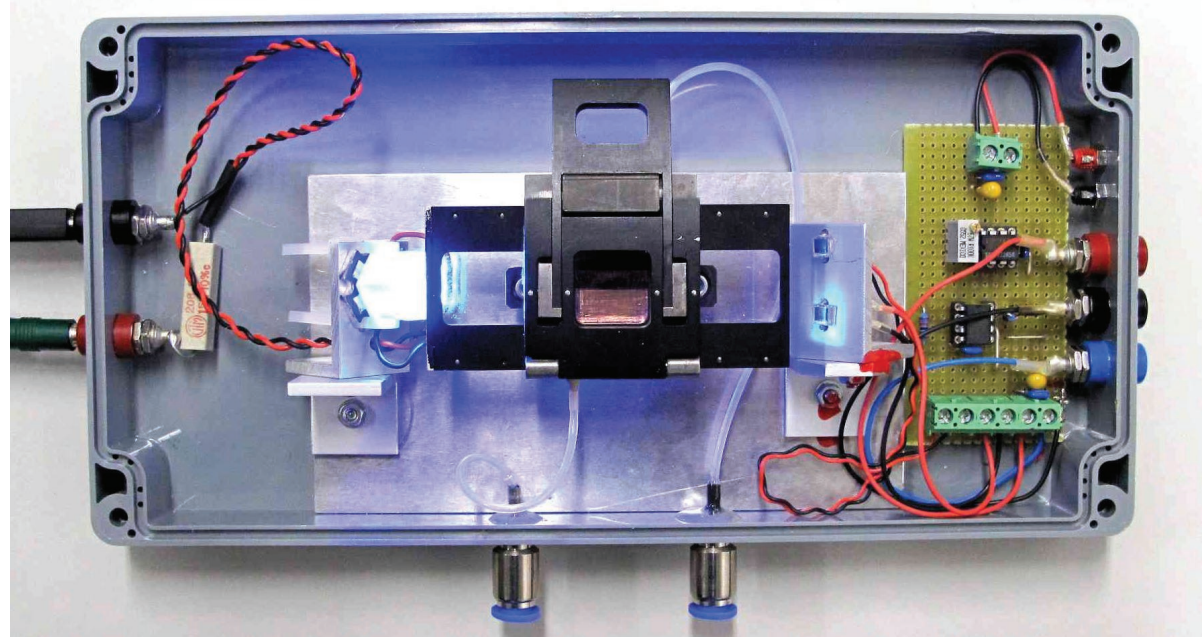

Figure 2: Set-up with gas cell for the gas measurements in a waveguide configuration.

\section{Colorimetric ammonia sensor}

Ammonia is for example produced during the putrefaction of perishable goods like seafood, and is present in chemical compounds such as explosives or fertilizers. Colorimetric detection of ammonia might be performed by different types of chemochromic reagents such as porphyrin-based or $\mathrm{pH}$ indicator-based films. We present the evaluation of colorimetric films for the detection of ammonia by using $\mathrm{pH}$-indicators. They were dissolved in a proper amount of solvents to obtain spin-coatable sub$\mu \mathrm{m}$ thin films. They have been successfully deposited onto glass waveguides to evaluate their optical properties and gas sensing operation. The influences of the matrix composition and the $\mathrm{pH}$ indicator 
used on their performances to detect ammonia is shown in Figure 3. All films exhibited an excellent selectivity towards $\mathrm{NH}_{3}$. A matrix composed of bromophenol blue and PMMA exhibited the best properties. Concentrations as low as $0.25 \mathrm{ppm}$ of $\mathrm{NH}_{3}$ were easily measured and the theoretical limit of detection was evaluated to $2 \mathrm{ppb}[2]$.

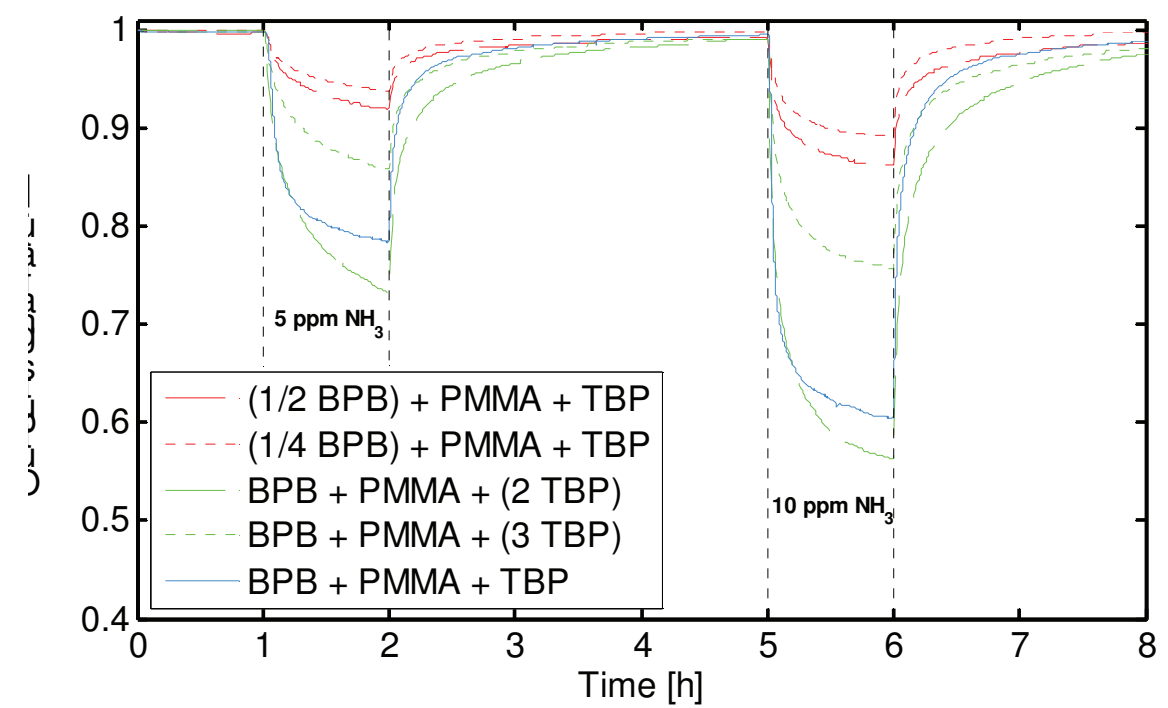

Figure 3: Gas measurements with the different films coated on the planar optical waveguide under 5 and $10 \mathrm{ppm}$ of $\mathrm{NH}_{3}$ in synthetic air with $50 \% \mathrm{RH}$ [2].

\section{Colorimetric carbon monoxide sensor}

For fire detection, a reliable detection of carbon monoxide is of high interest. The first notification of colorimetric detection of $\mathrm{CO}$ by diode pentoxide was in 1928 [3]. Other possible gasochromic materials are complexes. The work presented here focuses on the investigations of a rhodium complex and its gasochromic behavior. The reaction scheme to $\mathrm{CO}$ is shown in Figure 4. The chemical formula of this complex is cis - $\left[\mathrm{Rh}_{2}\left(\mathrm{C}_{6} \mathrm{H}_{4} \mathrm{PPh}_{2}\right)_{2}\left(\mathrm{O}_{2} \mathrm{CCH}_{3}\right)_{2}\right]\left(\mathrm{HO}_{2} \mathrm{CCH}_{3}\right)_{2}\left(\right.$ short: $\left.1^{*}\left(\mathrm{CH}_{3} \mathrm{CO}_{2} \mathrm{H}\right)_{2}\right)$. The $\mathrm{CO}$ is bound in axial direction under release of two acetic acids [5].

This complex is not commercially available. Therefore it was synthesized from its single components rhodium acetate, triphenylphosphine and acetic acid according to [4]. All components were obtained from Sigma Aldrich.

The time-dependent color change in solution due to a CO expose of $300 \mathrm{ppm}$ is shown in Figure 5 , the corresponding transmission spectra in Figure 6 . The dye changes from violet to yellow. The color change is completely reversible. In case of the sensor performance as fire detector, reversibility is very important. A waveguide-based measurement is shown in Figure 7. The synthesized dye was embedded into a PVC matrix with tetrahydrofuran (THF) as solvent and deposited onto a microscopic slide. The measurements were performed with the measurement chamber shown in Figure 2 and a blue LED. The sensor was exposed to $100 \mathrm{ppm}$ for 100 minutes. The measurement shows that the sensor reaction is completely reversible. 


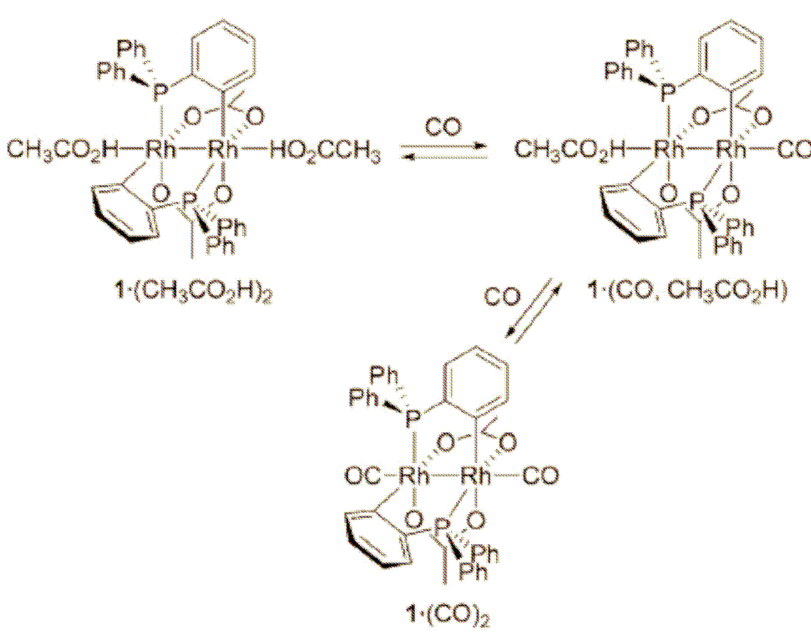

Figure 4: Reaction scheme of carbon monoxide and rhodium complex $1^{*}\left(\mathrm{CH}_{3} \mathrm{CO}_{2} \mathrm{H}\right)_{2}$ [4].

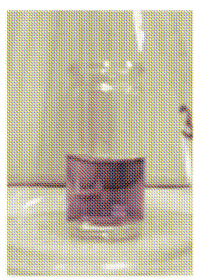

(a) Start

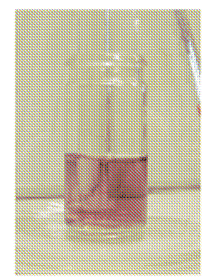

(b) $2.5 \mathrm{~min}$

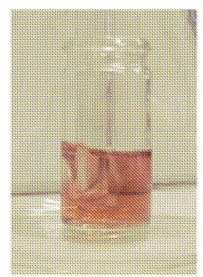

(c) $8,5 \mathrm{~min}$

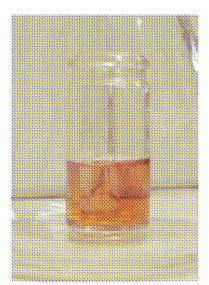

(d) 11,5 min

Figure 5: Reaction of $5 \mathrm{mg} 1 \cdot(\mathrm{CH} 3 \mathrm{CO} 2 \mathrm{H}) 2$ dissolved in $3,06 \mathrm{ml}$ chloroform during the exposure $300 \mathrm{ppm} \mathrm{CO}$. The color change is very fast and completely reversible (not shown in this picture).

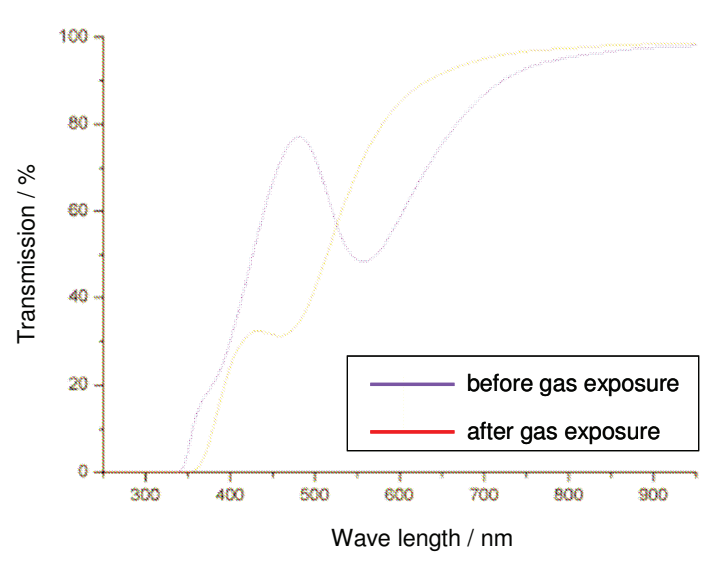

Figure 6: UV/VIS transmission spectra of $1 \cdot\left(\mathrm{CH}_{3} \mathrm{CO}_{2} \mathrm{H}\right)_{2}$ before and after exposure to $100 \mathrm{ppm} \mathrm{CO}$. The color of the sample changes from violet to yellow. 


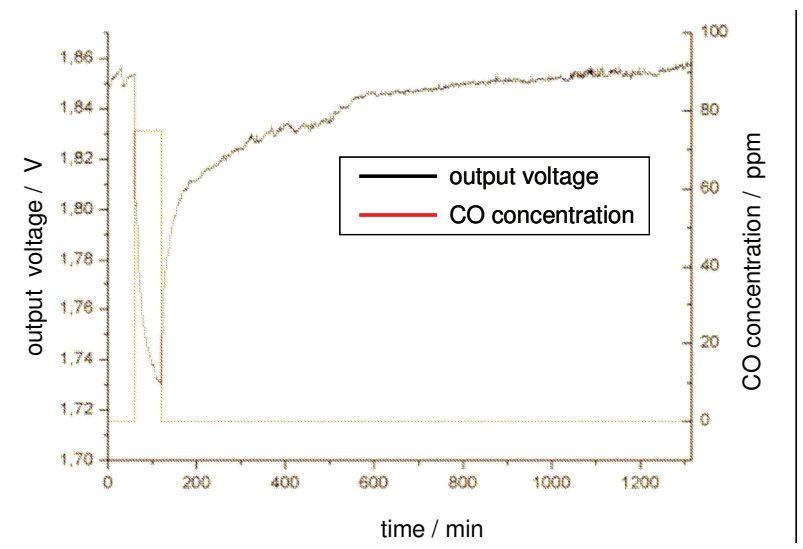

Figure 7: Waveguide-based colorimetric gas measurement of a rhodium complex exposed to $100 \mathrm{ppm} \mathrm{NO}_{2}$ for 100 minutes. The color change is completely reversible.

\section{Colorimetric nitrogen dioxide sensor}

Possible chromogenic materials for $\mathrm{NO}_{2}$ detection are metallo-porphyrines. Porphyrins are pigments with characteristic colors and are widely used in industrial applications. Metalloporphyrins consist of four pyrrole rings connected via methins, with a centered metal atom, e.g. zinc, iron or cobalt. Being part of many proteins, metallo-porphyrines have various functions in biological systems. The porphyrin heme is part of hemoglobin and manages the oxygen transport and storage in the circulatory system [6,7].

We focused on the investigation of (5,10,15,20-tetraphenylporphyrin)-Zinc (ZnTPP) as chromogenic material for $\mathrm{NO}_{2}$ detection. ZnTPP has a characteristic purple color and is soluble in a small amount of THF. For gas measurements, the ZnTPP is embedded into a PVC matrix. The $\mathrm{NO}_{2}$ reaction is a complex reaction with the centered Zn-atom [8]. The gas-dependent changes in the UV/VIS spectra are shown in measurement Figure 8. The color change from purple to yellow occurs in the blue spectral range.

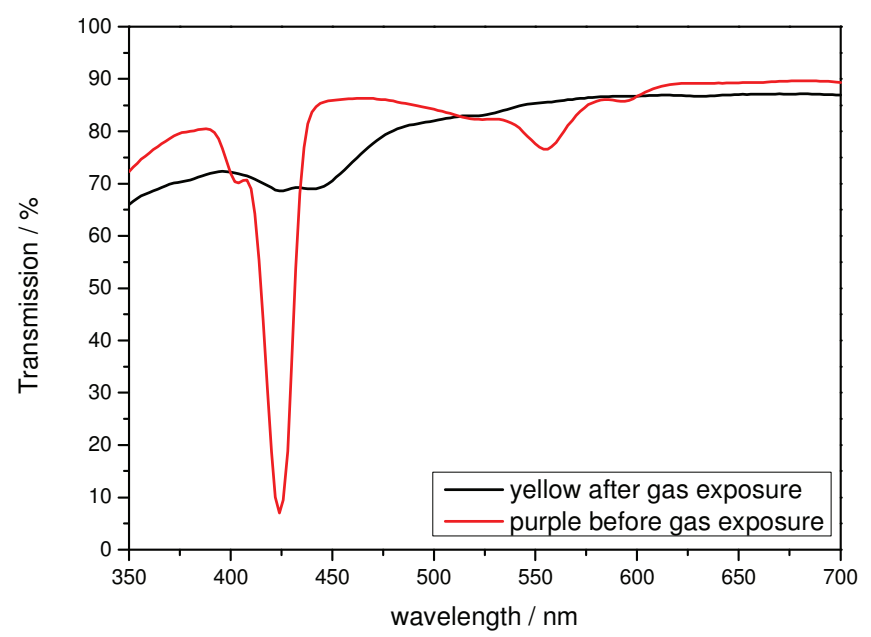

Figure 8: Transmission spectra of ZnTPP before and after reaction to $5 \mathrm{ppm} \mathrm{NO}$. The color change from purple to yellow is located in the blue spectral range.

An important indicator of changes in sample color is the change in the processed gas concentration. These changes are ascertainable with Beer-Lamber's law. The change of the absorption coefficient at $450 \mathrm{~nm}$ is shown in Figure 9. One main feature of colorimetric gas sensors is their high selectivity to only one gas. To investigate the selectivity of ZnTPP to only NO2, the sensor was also exposed to ammonia, ethanol, carbon monoxide and carbon dioxide. No cross sensitivities were detectable. 


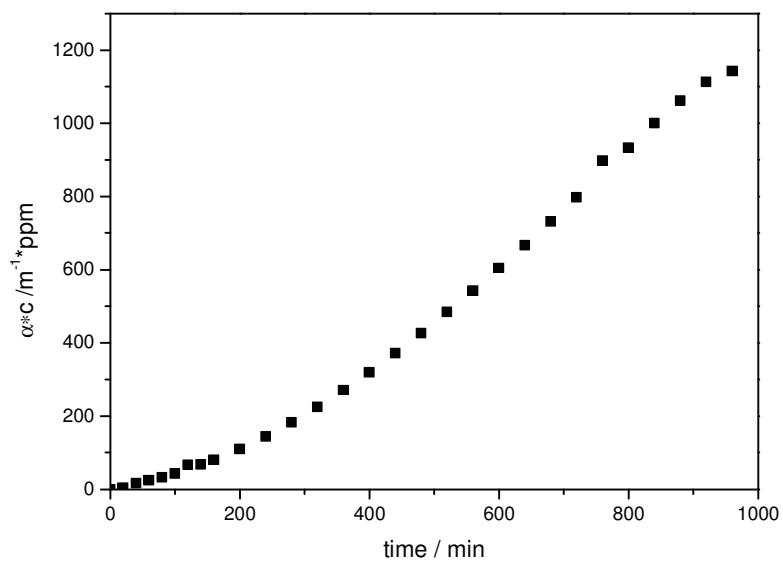

Figure 9. Changes in the transmission due to processed $\mathrm{NO}_{2}$ at $450 \mathrm{~nm}$.

\section{Summary and Outlook}

In this work we have shown different possibilities for gasochromic gas sensors. The work focused on the detection of ammonia, carbon monoxide and nitrogen dioxide. The detection limit for ammonia is $5 \mathrm{ppm}$, for $\mathrm{CO} 30 \mathrm{ppm}$ and for $\mathrm{NO}_{2} 0.5 \mathrm{ppm}$. The color dyes are embedded into different polymeric matrices. Remarkably are the low detection limits and the selectivity to only one target gas. The new waveguidebased measurement principle offers the possibility to develop selective and low power gas sensors.

\section{Literature}

[1] www.dräger.com, website available at 28.Feb.10011

[2] J. Courbat, D. Briand, J. Damon-Lacoste, J. Wöllenstein, N.F. de Rooij "Evaluation of pH IndicatorBased Colorimetric Films for Ammonia Detection Using Optical Waveguides", Sensors and Actuators B Chemical, 2009

[3] M. J. Martinek and W. C. Marti, "Modified iodine-pentoxide method for determination of carbon monoxide in air and blood" American Journal of Public Health, 1928, 293-298

[4] Cotton et. al. "Structural and electrochemical characterization of the novel orthometalated dirhodium(II) compounds Rh2(O2CMe)2[Ph2P(C6H4)]2·2L", Organometallics, 1985, 8-13

[5] Esteban et. al. "Sensitive and Selective Chromogenic Sensing of Carbon Monoxide by Using Binuclear Rhodium Complexes" Angewandte Chemie, Volume 122, 2010, 5054-5057

[6] T. Matsui, M. Unno, M. Ikeda-Saito, "Heme Oxygenase Reveals Its Strategy for Catalyzing Three Successive Oxygenation Reactions", Acc. Chem. Res. 43 (2), 2010, 240-247

[7] D. Mansuy, "A brief history of the contribution of metalloporphyrin models to cytochrome P450 chemistry and oxidation catalysis", Compt. Rend. Chim. 10 (4-5), 2007, 392-413

[8] D. Filippini, "Chemical Sensing with Familiar Devices”, Angew. Chem. Int. Ed. 45, 2006, 3800-3803 